\title{
mujeres supervivientes de violencia de pareja: factores y procesos psicológicos implicados en la decisión de permanecer 0 abandonar la relación
}

\author{
Survivors of Partner Uiolence: Factors and Psychological Processes Involved in the \\ Decision to Stay or Leave the Relationship \\ mulheres sobreviventes de violência de seus parceiros: fatores e processos \\ psicológicos implicados na decisão de permanecer ou abandonar a relação
}

\author{
Jesús Joel Aiquipa Tello* \\ Nelly María Canción Suárez \\ Institución Vida Mujer
}

Doi: https://doi.org/10.12804/revistas.urosario.edu.co/apl/a.7139

\begin{abstract}
Resumen
Entre todos los contextos de violencia a la mujer, la violencia de pareja es la más frecuente a nivel mundial. Esta genera consecuencias perjudiciales para la mujer, la familia y la sociedad. En el ámbito científico, denodados esfuerzos se realizan para identificar aquellos factores asociados a este tipo de violencia. Así, es importante indagar aquellas razones referidas por las mismas protagonistas para continuar o terminar una relación violenta, pues tales motivos pueden asumir la función de factores subjetivos determinantes de su conducta. El objetivo del estudio fue identificar y comprender los factores y procesos psicológicos que influyeron en la decisión de una mujer para continuar o terminar una relación de pareja violenta. Mediante una metodología cualitativa y un diseño de teoría fundamentada, se realizaron entrevistas individuales y dos sesiones de grupo
\end{abstract}

focal a nueve mujeres adultas residentes en Lima, Perú, sobrevivientes de una relación de pareja heterosexual violenta. Los datos se analizaron mediante un proceso de comparación constante. Los resultados indican que factores como la dependencia emocional, la tendencia protectora y el sistema de creencias irracionales influyeron en las participantes para continuar la relación violenta. Por su parte, factores como la priorización del bienestar de los hijos y el apoyo social movilizaron procesos psicológicos de afrontamiento para terminarla. Estos resultados muestran el papel activo que asume el grupo de mujeres para enfrentar la violencia de pareja. Los hallazgos deben considerarse en la implementación de programas de tratamiento y prevención, a fin de optimizar la calidad de la atención en los servicios de salud. Palabras clave: violencia de pareja, mujeres supervivientes, procesos psicológicos, afrontamiento.

* Dirigir correspondencia a Jesús Joel Aiquipa Tello: Institución Vida Mujer, Lima, Perú. Correio eletrônico: jesus.aiquipat@ gmail.com

Como citar este artículo: Aiquipa Tello, J. J., \& Canción Suárez, N. M. (2020). Mujeres supervivientes de violencia de pareja: factores y procesos psicológicos implicados en la decisión de permanecer o abandonar la relación. Avances en Psicología Latinoamericana, 38(1), 189-202. https://doi.org/10.12804/revistas.urosario.edu.co/apl/a.7139 


\section{fbstract}

Among all the contexts of violence against women, intimate partner violence is the most frequent worldwide, generating harmful consequences for women, family, and society. In the scientific field, strenuous efforts are made to identify those factors associated with this type of violence. Thus, it is essential to investigate those reasons referred by the same protagonists to continue or end a violent relationship, since such can assume the function of subjective factors that determine their behavior. The objective of the study was to identify and understand the psychological factors and processes that influenced the woman's decision to continue or end a violent relationship. Using a qualitative methodology and a grounded theory design, individual interviews and two focus group sessions were conducted with nine adult women survivors in Lima, Peru, of a heterosexual violent relationship. The data were analyzed through a constant comparison process. The results indicate that factors such as emotional dependence, protective tendency, and irrational belief system influenced the participants to continue the violent relationship, while factors such as prioritization of children's welfare and social support mobilized psychological coping processes to end the relationship. These results show the active role assumed by the group of women to face partner violence; the findings should be considered in the implementation of treatment and prevention programs to optimize the quality of care in health services.

Keywords: Couple violence, surviving women, psychological processes, coping.

\section{Resumo}

Entre todos os contextos de violência à mulher, a violência de seus parceiros é a mais frequente no nível mundial, gerando consequências prejudiciais na mulher, família e sociedade. No âmbito científico, denodados esforços se realizam para identificar aqueles fatores associados a este tipo de violência. Assim, é importante indagar aqueles motivos referidos pelas mesmas protagonistas para continuar ou terminar uma relação violenta, pois tais motivos podem assumir a função de fatores subjetivos determinantes de sua conduta.
O objetivo do estudo foi identificar e compreender os fatores e processos psicológicos que influíram na decisão da mulher para continuar ou terminar uma relação violenta. Através de uma metodologia qualitativa e um desenho de teoria fundamentada, se realizaram entrevistas individuais e duas sessões de grupo focal a nove mulheres adultas residentes e, Lima, Peru, sobreviventes de uma relação heterossexual violenta. Os dados se analisaram através de um processo de comparação constante. Os resultados indicam que fatores como a dependência emocional, tendência protetora e sistema de crenças irracionais influíram nas participantes para continuar a relação violenta; enquanto que fatores como priorização do bem-estar dos filhos e apoio social mobilizaram processos psicológicos de afrontamento para terminar a relação. Estes resultados mostram o papel ativo que assumem o grupo de mulheres para enfrentar a violência de seus parceiros e os resultados devem considerar-se na implementação de programas de tratamento e prevenção com o objetivo de otimizar a qualidade de atenção nos serviços de saúde.

Palavras-chave: violência de seu parceiro, mulheres sobreviventes, processos psicológicos, afrontamento.

La violencia de pareja se define como cualquier comportamiento dentro de una relación íntima que causa daño físico, psicológico o sexual a sus miembros. Tal comportamiento incluye agresión física, abuso psicológico, relaciones sexuales forzadas y comportamientos de control (Heise, \& García-Moreno, 2003), y abarca la violencia infligida por los cónyuges y los compañeros actuales y anteriores. Es importante señalar que la mayoría de quienes experimentan violencia de pareja son mujeres, y son los hombres quienes la perpetran (United Nations, 2015).

La violencia por parte de la pareja genera consecuencias perjudiciales físicas, psicológicas, sociales y económicas no solo en la mujer, sino también en su grupo familiar y en la sociedad en general (Walker, 2017; Watkins et al., 2014; World Bank Group, 2015). Así, estadísticas internacionales reportan que 
este tipo de violencia afecta alrededor del $30 \%$ de las mujeres en todo el mundo (Organización Panamericana de la Salud [OPS], 2014; United Nations, 2015). En el Perú, la Encuesta Demográfica y de Salud Familiar realizada por el Instituto Nacional de Estadística e Informática (INEI, 2017) indica que el $68.2 \%$ de las mujeres alguna vez emparejadas sufrieron algún tipo de violencia por parte del esposo o compañero. Los actos violentos pueden durar años e ir agravándose hasta el punto de terminar en asesinato de la mujer.

Aquellas mujeres que logran reponerse a una situación de violencia de pareja, sobre todo a las tentativas de asesinato, son consideradas mujeres supervivientes, pues son personas que pudieron conservar su vida después de los continuos y crueles actos de violencia. Por supuesto, este desenlace es un proceso complejo que implica la ocurrencia de diversos factores, algunos de los cuales influyeron para que continúe la relación, mientras que otros facilitaron la terminación de esta.

En el estudio de la violencia a la mujer por la pareja es importante considerar los motivos o razones referidas por las mismas protagonistas para continuar o terminar una relación violenta. Tales motivos pueden asumir la función de factores en la medida que refieren procesos o situaciones que influyen en su decisión. El estudio de esos motivos, razones o factores desde la perspectiva y vivencias de las mujeres violentadas tiene un aporte invaluable para profundizar el tema, pues con ello puede entenderse cómo ellas mismas toleraron el maltrato en una etapa de su vida mientras que en otra, decidieron poner fin a los maltratos y salieron victoriosas de ese recorrido cuando muchas no lo logran.

Al respecto, se reportan algunos factores psicológicos y circunstanciales asociados al inicio y mantenimiento de una relación de pareja violenta. Pueden advertirse así rasgos de personalidad compulsivos en las mujeres víctimas (Ballester, \& Villanueva, 2014); dependencia emocional a la pareja (Aiquipa, 2015); creencias y esquemas cognitivos disfuncionales (Hayati et al., 2013; Huerta et al., 2016; Nóblega, \& Muñoz, 2009); indefensión aprendida (Walker, 2017); estrategias ineficaces de afrontamiento como aislamiento y autoinculpación (Molina, \& Moreno, 2015); déficit en autoestima (Reich et al., 2015; Ocampo, 2015); autoeficacia disminuida (Tirone, Shorey, Nathanson, \& Rhatigan, 2014); no tolerancia de emociones disfóricas como culpa, vergüenza y miedo (Puente-Martínez, Ubillos-Landa, Echeburúa, \& Páez-Rovira, 2016), y falta de redes de apoyo (Molina, \& Moreno, 2015).

Si bien es lógico pensar que una mujer con algunos de los factores señalados puede continuar con la pareja agresora, recibiendo niveles cada vez más graves de maltrato - incluso hasta ser asesinada-, existen casos en que mujeres con esos factores deciden finalizar la relación. Asimismo, esta decisión no necesariamente es posterior a recibir algún tipo de intervención psicológica, sino que puede presentarse en mujeres que por diferentes circunstancias no pudieron acceder a ese tipo de ayuda. Si bien la intervención psicológica puede promover y acelerar la toma de decisiones, debe haber algunos factores que interactúan para que la mujer decida poner fin a los actos de violencia incluso en ausencia de intervención profesional. Estos factores probablemente propician que la mujer asuma una actitud diferente y emita respuestas de afrontamiento, distintos a los que venía realizando, para conseguir así separase del agresor.

En este sentido, algunos estudios señalan factores que facilitan la toma de decisiones de una mujer para terminar una relación abusiva. Así, se encuentran variables como evaluaciones cognitivas y estilo de afrontamiento comprometido (Fleming, \& Resick, 2017); apoyo psicológico, perseverancia, espiritualidad y responsabilidad (Zalapa, García, \& Figueroa, 2012); apoyo social (Buesa, \& Calvete, 2013; Montero, Martín-Baena, Escribà-Agüir, Vives-Cases, \& Ruiz-Pérez, 2015; Shanthakumari, Chandra, Riazantseva, \& Stewart, 2014); bienestar de los hijos, en aquellas mujeres que los tienen (Hayati et al., 2013; OPS, 2013; Shanthakumari et 
al., 2014; Rasool, 2016), y soporte social (Jiménez, Blanco, Medina, \& Gómez, 2013), laboral y económico (Alsaker, Moen, Baste, \& Morken, 2016; Nóblega, \& Muñoz, 2009).

Otros estudios reportan las consecuencias psicológicas de terminar una relación de pareja violenta. En este sentido, algunas mujeres pudieron comprender que tenían una fuerza interna que las ayudó a sobrevivir y moverse a través del trauma (Matheson et al., 2015), reconstruyeron su identidad y recuperaron su libertad y poder para tomar decisiones sobre sus propias vidas (Flasch, Murray, \& Crowe, 2017). En congruencia con lo expuesto, el objetivo de la investigación es identificar, describir y comprender, desde la perspectiva de las mujeres supervivientes, aquellos factores y procesos implicados en la decisión para continuar o terminar una relación de pareja violenta.

Gran parte de la investigación sobre el tema estudia los factores que mantienen la violencia, enfocándose básicamente en variables sociodemográficas. Algunos otros indagan aquellas situaciones que facilitan el decidir terminar la relación violenta. Sin embargo, son pocos los estudios que abordan la influencia que ambos tuvieron en distintos momentos de la relación, sus interacciones, procesos y el impacto de esa decisión en la mujer, desde la perspectiva de las protagonistas. Enfocar así estos procesos permite revalorar el papel activo que la mujer tiene en el afrontamiento de este problema. Asimismo, comprender estos aspectos permitirá efectivizar las atenciones de los operadores de justicia, mejorar las propuestas de tratamiento de profesionales de la salud y tomar acciones preventivas.

\section{Método}

\section{Tipo de investigación y diseño}

Estudio empírico con metodología cualitativa, que se orienta a generar conocimiento científico basándose en un proceso constructivo interpretativo entre participantes e investigadores. El diseño empleado se basa en la teoría fundamentada (Glaser, \& Strauss, 1967), pues se pretende identificar y comprender, desde la perspectiva y las vivencias de las colaboradoras, los factores (motivos) y procesos psicológicos que influyeron en ellas para continuar o terminar una relación violenta.

\section{Participantes}

Se seleccionaron las colaboradoras de acuerdo con criterios no probabilísticos, concretamente casos tipo (Flick, 2015), congruente con el enfoque de estudio. Respecto al número de participantes, se tomó en consideración el poder de información (Malterud, Siersma, \& Guassora, 2016), el cual considera aspectos diferentes a la cantidad, tales como objetivo del estudio, especificidad de la muestra y calidad del diálogo. Así, las colaboradoras fueron en total nueve mujeres entre 28 y 53 años, residentes en la ciudad de Lima, con nivel socioeconómico bajo y medio, que vivieron una relación violenta de pareja heterosexual en los últimos tres años. Cinco de ellas tienen nivel de instrucción superior técnica incompleta, una superior universitaria incompleta y tres culminaron la secundaria. Durante la realización del estudio, todas las participantes tenían una ocupación laboral, ya fuera estable (5 mujeres) o trabajo eventual (4 participantes). Si bien todas las colaboradoras estuvieron separadas de sus parejas durante la recolección de datos, tres de ellas aún tienen la condición de casadas. Todas las participantes tienen hijos menores de edad, varones y mujeres, cinco tienen 1 hijo y las cuatro restantes entre 2 a 4 hijos. La edad promedio en la que iniciaron su relación de pareja con el agresor fue a los 22 años. Desde el inicio de la relación hasta la primera vez que recibieron los primeros maltratos psicológicos y físicos pasaron en promedio $12 \mathrm{me}-$ ses. El tiempo que las participantes toleraron los episodios de violencia es variable y oscila entre 2 y 15 años, los episodios se dieron de violencia de 
forma intermitente. Asimismo, el tiempo promedio que estuvieron separadas de la pareja hasta la fecha de participación en el estudio fue de un año y medio.

\section{Técnicas de recolección de datos}

Se empleó la entrevista individual, dado que la naturaleza del fenómeno sugería el empleo de un recurso que facilitara la cercanía con la participante, pues los aspectos abordados son personales. Así, la entrevista permite representar la visión de la persona de manera justa y presentarla coherentemente con sus significados (Miller, \& Glassner, 2016). De esta forma, se realizaron en total nueve sesiones de entrevistas individuales (identificadas con los códigos E1, E2, E3, E4, E5, E6, E7, E8 y E9).

Asimismo, se empleó el grupo focal (GF), puesto que este recurso permite que las participantes expresen y compartan con sus compañeras emociones, pensamientos, preocupaciones, expectativas y otros tópicos respecto a la violencia de pareja. Se realizaron dos sesiones de grupos focales (codificadas como GF1 y GF2). Estas sesiones se realizaron con cinco de las nueve participantes. Para ellas se utilizó el mismo contenido de la guía de entrevista individual, con las modificaciones necesarias para el grupo.

Tanto para la entrevista individual como para el grupo focal se usó como instrumento una guía de entrevista con los siguientes ejes temáticos: circunstancias del inicio del maltrato, tipos de maltrato recibidos, atribuciones del maltrato, impacto del maltrato en la usuaria y en su familia, motivos para dejar la relación violenta y situación actual de la usuaria. Se registró la información mediante un grabador de voz digital.

\section{Procedimiento}

Luego de elaborar la guía de entrevista, se coordinó con una colaboradora (agente clave), quien trabaja en la municipalidad de un distrito limeño y tenía los contactos con las potenciales participantes del estudio. A través de ella, se pudo acceder a cuatro mujeres. Otras cinco participantes fueron convocadas por la institución a través de uno de los programas de tratamiento. Las entrevistas individuales y el GF se realizaron en la institución, en un ambiente cerrado con las condiciones necesarias y adecuadas para garantizar su comodidad y facilitar el diálogo. Las entrevistas individuales se realizaron en una sesión con un tiempo de duración entre 45 a 65 minutos. El GF se realizó con cinco de las participantes, en dos sesiones y con un tiempo promedio de 110 minutos por sesión. En todos los casos se informó sobre el objetivo del estudio, se solicitó el consentimiento informado por escrito y se grabaron las entrevistas en audio. La información se recolectó durante 8 meses (noviembre de 2016 a junio de 2017). Terminada la recolección de información, se procedió a transcribir los audios grabados para su procesamiento.

\section{Procesamiento de datos}

Para analizar la información, se utilizó el método de la teoría fundamentada (Glaser, \& Strauss, 1967), que permite desarrollar análisis teóricos inductivos a partir de los datos recopilados mediante un proceso de comparación constante (Silverman, 2015). En este sentido, tomando en cuenta las estrategias sugeridas por Rapley (2016), la información fue segmentada por unidades temáticas. Luego se realizó la categorización de primer nivel, a fin de encontrar categorías relevantes para el estudio. A dichas categorías se les asignó un código y se hizo la codificación a todo el material. Para encontrar categorías más interpretativas, se realizó la categorización de segundo nivel, para lo cual se compararon las categorías iniciales. De forma paralela, se relacionaron las categorías finales entre sí para obtener temas principales y patrones, a fin de elaborar modelos comprensivos sobre el fenómeno estudiado. Se usó el programa informático Atlas.ti 7.16 como apoyo para el análisis cualitativo. 


\section{Aspectos éticos}

El estudio cumple con aspectos éticos congruentes con el Código de Ética y Deontología del Colegio de Psicólogos del Perú (2018). Asimismo, se siguieron los lineamientos éticos propuestos por el Ministerio de la Mujer y Población Vulnerable para las investigaciones en violencia familiar y sexual (Bardales, 2014). Se solicitó el consentimiento informado escrito a las participantes, se explicó la naturaleza del estudio, su objetivo, los fines de la información recabada, la confidencialidad de los datos, el respeto al anonimato y la posibilidad de abandonar la investigación cuando lo consideren pertinente.

\section{Criterios de calidad}

Para garantizar la calidad y rigurosidad del estudio, se emplearon estrategias de promoción de calidad (Flick, 2014) durante el proceso de indagación. Así, para cumplir con el criterio de credibilidad, se utilizó un grabador de voz digital, para registrar las respuestas de las participantes tanto en las intervenciones individuales, como grupales. Con ello, la información recolectada, su procesamiento y la recuperación de unidades de análisis se sustentan en las vivencias de las participantes tal como ellas lo manifestaron. La dependencia del estudio se concretó a través de la triangulación de técnicas de recolección de datos (entrevistas individuales y GF), aplicación de instrumentos con el mismo contenido temático, tiempo prolongando de interacción con las participantes y similitud de condiciones en la aplicación de instrumentos. La transferibilidad se sustenta en la descripción detallada de las características de las participantes y del proceso de recolección de información. Para garantizar la confirmabilidad del estudio, se utilizó como estrategia la reflexibilidad, sesiones de discusión por parte del equipo para desarrollar una conciencia crítica para controlar cuidadosamente el impacto de sus prejuicios, creencias y experiencias personales durante el proceso de investigación
(Berger, 2015). Así, se realizaron tres sesiones de reflexión, dos después de recabar la información y una durante el análisis de esta.

\section{Resultados}

\section{Modalidad de violencia de pareja sufrida}

Las participantes recibieron maltrato psicológico como prohibiciones sobre su vestimenta y posibilidad de trabajar, cuestionamientos sobre su dignidad como mujer y madre, gritos, humillaciones, burlas hacia su persona, insultos frecuentes y amenazas. También sufrieron violencia física como jalones de cabello, empujones, puñetes (en rostro, cabeza y cuerpo), patadas, golpes con objetos e intentos de homicidio ("mi pareja intentó asesinarme en tres oportunidades", E7). El maltrato patrimonial o económico consistió básicamente en disminución o retiro del sustento económico para ella y los hijos en común. Tres de las participantes manifestaron que sufrieron violencia sexual por parte de su pareja:

\footnotetext{
"Tras pasar los años, me abusaba sexualmente mientras yo dormía. Tenía el sueño pesado y en varias ocasiones me desperté y me sentía mojada" (E1).

"Él me exigía tener relaciones sexuales... Las relaciones sexuales que teníamos eran en contra de mi voluntad, prácticamente abusaba de mí, y fue así que me embarace de la menor de mis hijas" (E2).

"Me obligaba hacer otras formas de relación sexual..., me daba asco" (E9).
}

\section{Consecuencias de la violencia de pareja en la mujer}

Si bien la violencia de pareja generó en las participantes secuelas físicas observables, como cicatrices y hematomas, son las consecuencias psicológicas, sobre todo las que implican la dimensión emocional, las que son percibidas por las participantes como las 
más perjudiciales. Así, se encontró que los malos tratos generan emociones disfóricas como ansiedad, tristeza e ira, emociones que se presentan muchas veces de forma simultánea: "Me sentía tensa, preocupada y empezaba a ponerme agresiva y le respondía; siempre estaba nostálgica y pensativa; paraba enojada" (E8). Se encontró además ansiedad y temor por ser agredida una vez más o perder la vida a manos de la pareja: "no puedo vivir tranquila, me siento nerviosa, con miedo" (E7).

También se hallaron sentimientos de culpa: "llegué a sentirme culpable y pensé que él cambiaría" (E8); autodesvalorización: "mi autoestima estaba en los suelos, no solía salir de casa ni recibir a ninguna de mis amistades" (E4); percepción de aislamiento social: "llegué a tener pensamientos de encierro, de estar en una isla y desprotegida, sin tener quien me apoyara" (E2), e inseguridad: "me sentía insegura de mí, de lo que hacía” (E3).

\section{Factores que influyen en la continuidad de la relación}

Este patrón hace referencia a los factores y procesos psicológicos que influyeron en la decisión de la mujer de continuar con la relación de pareja violenta. Así, se identificaron creencias irracionales sobre ella misma, el amor y la pareja:

"Pensaba que no valía nada, que lo que decía no importaba" (E8).

"Creía que la violencia era algo normal. Creí que todas las parejas pasaban por eso, que no podría salir adelante sin él y que no era nada sin él” (E1).

Además de estas creencias irracionales, las participantes presentaron rasgos de dependencia emocional, definida como una necesidad de vinculación afectiva extrema hacia la pareja, principalmente necesidad de afecto y prioridad de la pareja:

"Me sentía controlado por él, primero era él, antes que mi papá, mi mamá, mi familia, primero era él. Cuando cocinaba, la mejor presa, el primer plato era para él. Si él no salía a algún sitio, yo no salía. Mi mundo era él" (GF2).

"A pesar de todo, lo amaba y seguí en una relación íntima con él. Me embaracé de mi segundo hijo" (E2).

"Mi amor por él era único, me entregué completamente, mi mundo giraba a su alrededor, empecé a alejarme de mi familia y mis amistades" (GF1).

La tendencia protectora, definida como la necesidad de ayudar y proteger a la pareja, incluso a costa de su integridad, parece ser un rasgo asociado a los rasgos de dependencia emocional y son efectos de creencias irracionales aprendidas:

"No he querido hacerle daño; pensaba que iba ir preso por eso no lo hacía, estaba cegada" (E3).

"Me avergüenza decir que nuevamente lo perdoné; se mostraba indefenso, cariñoso, comprensivo, pero luego se transformaba" (GF1).

"Me rogaba, me daba pena..., yo lo quería y me apenaba abandonarlo y dejarlo solo" (GF2).

"Lo perdoné en varias oportunidades, no quería perjudicarlo" (E7)

"Me daba pena, su familia no lo quería, no estaban con él, por eso lo perdonaba" (E9).

En estas condiciones, se encontró que la mujer tiende a atribuirse la responsabilidad de la relación y del comportamiento violento de la pareja, la justifica y niega su situación. Asimismo, tolera el maltrato y permanece en la relación violenta. Esta interacción de factores se muestra en la Figura 1 y queda ejemplificada en el relato de E1:

"Sentía que no podía alejarlo de mí y seguí a su lado. Llegó el momento en que me sentía dependiente de él, no quería dejarlo, sentía pena y tristeza si me alejaba. Me daba pena lo que él estuviera pasando ya que no tenía familia en Lima quien lo apoyara. Siempre fui consciente de que debía denunciarlo, pero en el fondo me preocupaba 
por él. Siempre lo justifiqué, pensé que cambiaría muchas veces, creí que se daría cuenta de todo el daño que le hacía a su familia, pero parecía que con él nada pasaba y que todo le daba igual.”

\section{Factores que influyen en la decisión de la mujer para terminar la relación de pareja}

La Figura 2 muestra aquellos factores situacionales o contextuales que activaron y movilizaron procesos psicológicos. Estos procesos promovieron la ejecución de estrategias de afrontamiento que facilitaron terminar la relación violenta. La priorización del bienestar de los hijos, entendida como la disposición psicológica que focaliza y orienta los procesos cognitivos, afectivos y conductuales para cuidar y proteger su integridad, constituye una de las razones compartidas y más relevantes para el grupo:

"Ahora he decidido hacer todo por la vía legal, ya no me importa más él. Lucharé para sacar adelante a mi hijo, él es mi motivo, mi lucha constante" (GF1).

"Eso fue lo más duro para mí; ya no era solo conmigo, ahora también era con mis hijos y no podía permitirlo. Yo les estaba pasando el sufrimiento de lo que yo estaba pasando. Con todo el amor del mundo decidí dejarlo hasta el día de hoy" (E3).

"Mis hijos son mi vida..., ahora soy más madre que mujer" (E6).

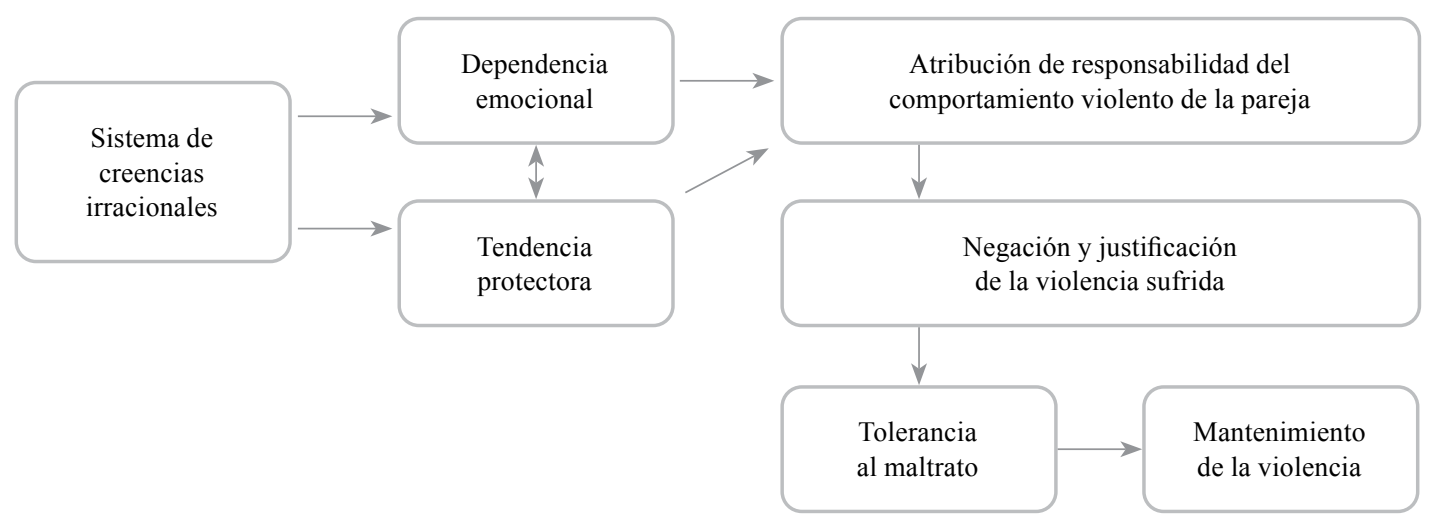

Figura 1. Factores influyentes en la decisión de continuar con la pareja agresora

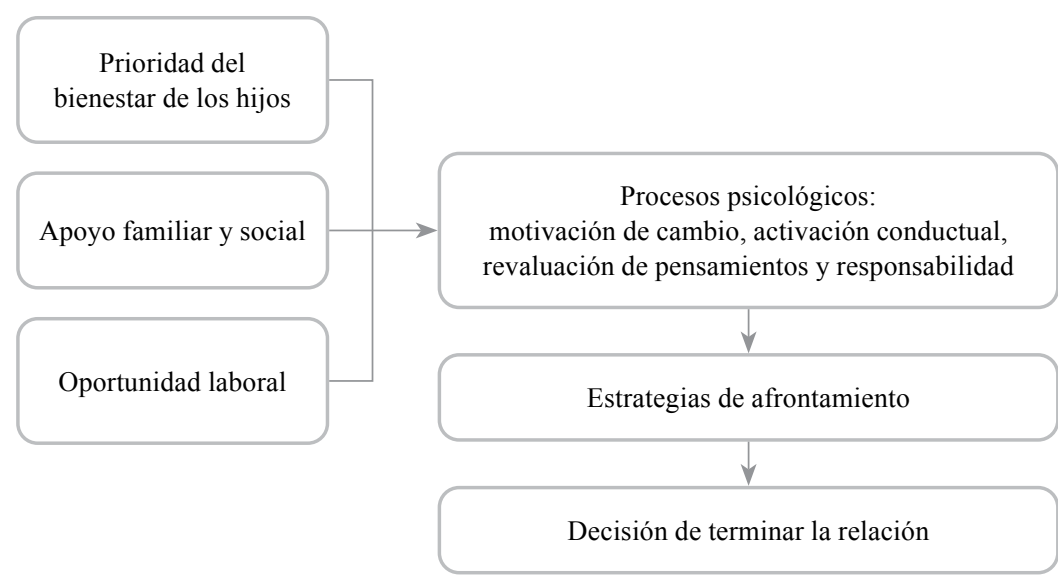

Figura 2. Factores que influyen en la decisión de terminar la relación de pareja 
El soporte o apoyo social que puede brindar la familia o las amistades, así como la oportunidad laboral, son factores que también facilitan la decisión de terminar la relación:

\footnotetext{
“Aunque pasado un tiempo mi madre se atrevió a denunciarlo, después de tantos años de sufrimiento" (E1).

"Tengo la suerte de tener a mis padres conmigo, me ayudan" (E5).

"Actualmente vendo pollo al por mayor con mis amistades, hago mis actividades, tengo vida propia, tengo tranquilidad, encamino mi vida, tengo paz, decido que hacer" (E6).
}

Factores como los mencionados movilizan procesos psicológicos como motivación de cambio, activación conductual y revaluación de pensamientos y responsabilidad, lo cual puede facilitar respuestas de afrontamiento, definidas como estrategias conductuales, cognitivas e incluso emocionales orientadas a distanciarse de la pareja, enfrentarlo o terminar la relación:

"Hasta hace un año me decidí dar por finalizada la relación. Me di cuenta que la violencia debía parar y tomé la decisión de dejarlo" (E1).

"Recién me di cuenta que alguien que te hace tanto daño no puede amarte" (E2).

"Vivía durante mucho tiempo muy agobiada; me armé de valor, decidí dar por terminada nuestra relación. Ahora, a través de la justicia, voy a hacer valer los derechos de mis hijas" (E3).

"Yo le dije a él, que se ubique porque yo con él no tengo nada, yo ya no vengo a ser nada de él" (E6).

\section{Beneficios percibidos tras terminar la relación de pareja violenta}

Cuando la mujer decide terminar la relación de maltrato, posiblemente transita por una eta- pa de duelo en la que experimenta sentimientos ambivalentes como tristeza, vergüenza, temor, cólera, tranquilidad y alivio. Esta etapa varía en tiempo e intensidad, pues depende de variables personales, el tipo vínculo afectivo y características de la violencia. Así, las participantes perciben como beneficiosas las consecuencias de su decisión:

"A raíz de que ya no estoy con él entendí que tener dignidad es lo más importante. Hoy en día me siento renovada, con muchas ganas de luchar y salir adelante, de emprender muchas cosas, aunque no me siento feliz con lo que hago, pero no lo menosprecio" (E3).

"Empezaba a darme cuenta que había perdido muchos años de mi vida. Ahora todo ha cambiado, soy muy feliz, mi hijo está estudiando en EE. UU., mis hijos me engríen, confían en mí" (GF2).

"Yo merezco ser feliz al lado de mi familia, de mis hijos, merezco ser feliz" (E7).

Las participantes perciben que su autoestima mejora, recuperan la esperanza y sentido de vida y se permiten reiniciar y disfrutar de actividades agradables y productivas que antes habían abandonado. Eventualmente, todo ello genera satisfacción consigo misma, sensación de logro y éxito que las motiva a ratificarse en su decisión.

"Yo tengo la fuerza suficiente para valerme por mí misma. Seguiré trabajando arduo, tengo muchas metas a futuro. Yo soy valiosa y saldré triunfadora de esto" (E4).

"Hoy mi vida ha dado una vuelta total, ahora tengo la libertad de decidir, hablar, hacer, comprarme lo que yo quiero; duermo con tranquilidad, puedo compartir con mis hijos, me siento libre de insultos" (E2).

"Yo estoy aprendiendo mucho por todo lo que he pasado, estoy estudiando un diplomado, hago amigos, conocer, porque no sabía nada" (E5). 


\section{Discusión de resultados}

El estudio analizó las vivencias, factores y procesos psicológicos que influyen en la decisión de la mujer para continuar una relación de pareja en la que hay violencia o terminarla. Es importante señalar que el contexto y las circunstancias específicas de cada una las participantes mediaron en las respuestas asumidas, alternándose en todos los casos ciclos de separación y retorno con la pareja agresora (Hayati et al., 2013).

Se identificó como factor mantenedor de la violencia el sistema de creencias que las participantes construyeron a lo largo de sus vidas, donde predominaban ideas erróneas sobre la relación de pareja, el amor, sí mismas y la violencia (Nóblega, \& Muñoz, 2009; Hayati et al., 2013; Huerta et al., 2016). Las continuas desvalorizaciones recibidas y las atribuciones que el agresor hacía sobre su comportamiento violento posiblemente facilitaron la asunción y confirmación de este tipo de creencias en las participantes. Además, al no tener un referente de contrastación de tales afirmaciones como consecuencia del aislamiento social impuesto o asumido por ellas mismas (Zalapa et al. 2012), construyeron creencias con contenido autodesvalorizador, que a su vez sustentan distorsiones cognitivas y repercuten en la percepción de autoeficacia (Matheson et al., 2015; Tirone et al., 2014).

Las participantes utilizaron estrategias cognitivas y conductuales para evitar el problema, negando, minimizando y justificando el maltrato. Específicamente, negar la violencia suele reportarse como una de las primeras respuestas (Messing, Mohr, \& Durfee, 2015). Esta estrategia reduce la angustia experimentada por el conflicto de sentirse comprometidas con la pareja y a la vez ser agredidas por él (Gilbert, \& Gordon, 2017). Así, encubren al agresor, mienten a sus familiares y conocidos haciéndoles creer que la relación es satisfactoria (Walker, 2017). Posiblemente, el realizar estas conductas sirve también para evitar la corroboración de algunas creencias centrales desadaptativas (e.g., “es horrible no ser amada"). Por tanto, la ejecución de aquellas estrategias representa una suerte de afirmación como personas, esposas, parejas o mujeres, que les permite salvaguardar su estima personal.

También se encontró que la dependencia emocional representa uno de los procesos psicológicos que puede influir en la mujer para perpetuar la relación, pues constituye un rasgo de personalidad disfuncional que las mantiene en la relación de maltrato (Amor, \& Echeburrúa 2010; Castelló, 2012; Huerta et al., 2016;). El miedo intenso a la posible ruptura, la prioridad que se confiere a la pareja y la actitud sumisa y subordinada hacia el otro, dimensiones propias de la dependencia emocional, propician comportamientos orientados a evitar romper la relación, que incluye tolerar conductas violentas (Aiquipa, 2015; Zalapa et al. 2012).

Pese a los continuos maltratos que sufrió la participante por parte de su pareja, aquella experimenta compasión de él cuando lo percibe vulnerable y solo. Le importa el bienestar de la pareja, no quiere lastimarlo, quiere protegerlo pues lo ve indefenso y arrepentido; en consecuencia, lo perdona. Al respecto, algunos estudios encontraron que niveles altos de satisfacción y compromiso experimentados en la relación de pareja, incrementaron la probabilidad de minimizar los maltratos, ser más indulgentes, perdonar a la pareja agresora y retomar la relación (Gilbert, \& Gordon, 2017). Esta tendencia protectora y la culpa que experimenta porque se atribuye la responsabilidad de la relación y del daño ocasionado, congruente con lo hallado por Molina y Moreno (2015), probablemente fomentan la continuidad de un vínculo que es perjudicial para ella y que no le permite terminar la relación.

Terminar la relación con la pareja agresora es un proceso que tiene más de un intento (Jiménez et al., 2013; Messing et al., 2015), ya sea por la actitud manipuladora de la pareja, por las características de la mujer o por los efectos psicológicos en ella producto de la violencia. Frente a esta situación, las participantes se mostraron ambivalentes, dudaban entre terminar la relación o continuar con 
ella y experimentaron tristeza, confusión, angustia, enojo y desconcierto, emociones que algunos autores refieren como parte de un proceso de duelo (Messing et al., 2015).

Para el grupo de participantes, la priorización del bienestar de los hijos representó el motivo más importante para decidir terminar la relación. La forma como repercutieron los episodios de maltrato en aquellos - quienes fueron en todos los casos testigos de la violencia-, así como los reclamos que estos les hicieron por tolerar la violencia, representó para las mujeres alicientes necesarios y suficientes para asumir su responsabilidad frente a dicha situación. Al respecto, la literatura señala que en los casos de mujeres que tienen hijos menores de edad, el bienestar de ellos es prioridad para aquellas y harán lo necesario para protegerlos (OPS, 2013; Hayati et al., 2013; Shanthakumari et al., 2014; Rasool, 2016).

El apoyo social, entendido como la disponibilidad de asistencia instrumental y emocional a través de familiares, amistades, conocidos e instituciones (Goodman, \& Smyth, 2011) posiblemente influye en el bienestar psicológico de las mujeres víctimas de maltrato y facilita terminar una relación violenta (Buesa, \& Calvete, 2013; Flasch et al., 2017; Shanthakumari et al., 2014; Montero et al., 2015). Al parecer, cuando la participante contrasta sus ideas sobre sí misma con el grupo social, esto facilita el proceso de evaluación de tales creencias, su veracidad y conveniencia, lo que posibilita el diálogo interno y confronta la normalización de los malos tratos como expresiones de una relación de pareja (Goodman, \& Smyth, 2011; Zalapa et al. 2012). Así también, se identificó la importancia de un soporte laboral económico. Como señalan algunos estudios, el laborar puede significar para las mujeres en esta situación un indicador de autoafirmación, pues se conciben útiles y productivas (Alsaker et al., 2016; Nóblega, \& Muñoz, 2009).

Parece ser que factores externos como los referidos permiten movilizar procesos psicológicos como motivación de cambio, activación conductual y revaluación de pensamientos y responsabilidad, que promueven estrategias de afrontamiento que aumentan la probabilidad de terminar la relación violenta. Así, los comportamientos y estrategias utilizadas por las participantes para sobrevivir a los episodios de violencia constituyen habilidades resilientes en las mujeres. En relación con este hallazgo, Shanthakumari et al. (2014) encontraron que las mujeres en situación similar emprenden estas estrategias como activación conductual, perseverancia, confrontación (con el agresor), confianza en sí mismas y evaluación reflexiva.

Si bien el proceso de terminar una relación de pareja implica la movilización de una serie de esfuerzos que generan agotamiento en las participantes, las consecuencias derivadas de tal decisión son percibidas por las mismas como liberadoras y gratificantes; recuperan así su valoración personal, autoeficacia, su propia identidad, libertad para realizar sus actividades y la toma de sus propias decisiones, aspectos encontrados también por Matheson et al. (2015) y Flasch et al. (2017). Jiménez et al. (2013), por el contrario, hallaron que las participantes de su estudio no consideraron una mejoría en su situación tras terminar la relación violenta. Aun así, pese a la historia de maltrato que tuvieron las participantes, posiblemente las sobrevivientes de una relación violenta consigan una salud óptima, actividad laboral, relaciones interpersonales satisfactorias y encontrar sentido a sus vidas que les genere bienestar (Flasch et al., 2017).

Contrario a lo que comúnmente se cree, las mujeres en situación de violencia continuamente están elaborando estrategias para minimizar la situación de maltrato. Incluso, la aparente condición de no "hacer nada" - frase muy empleada por operadores de justicia, familiares y amistades-, representa una estrategia para reducir la agresión por parte de la pareja. Posiblemente dichas estrategias parecen ser disfuncionales, pero son la mejor alternativa que las mujeres pudieron implementar para afrontar esa situación. En consecuencia, el estudio aporta información relevante para comprender el papel 
activo que asumen muchas mujeres para enfrentar una relación de pareja violenta, lo cual es una muestra del valor y fuerza que tienen para revertir situaciones complejas, incluso ante la adversidad.

Es pertinente señalar que la información obtenida corresponde a mujeres con determinadas características, y tal vez una de las más relevantes fue que todas tenían hijos menores de edad con las parejas agresoras, lo cual representa un aspecto medular cuando tomaron la decisión de terminar la relación. Esto debido al tipo de muestreo usado, pues conformaron parte del estudio usuarias voluntarias. Factores distintos a los encontrados pueden emerger al estudiar mujeres sin hijos que sufren violencia por parte de sus parejas.

Los resultados obtenidos constituyen una base teórica sustentada en los datos y la experiencia de las participantes, respecto a algunos factores y procesos que obstaculizan o facilitan el terminar una relación de pareja violenta en un grupo de mujeres. Esta información es potencialmente útil para la intervención en estos casos. Por ejemplo, tomando como referencia lo encontrado, las autoridades respectivas podrán capacitar y desarrollar en los operadores de justicia (efectivos policiales, fiscales, jueces) habilidades empáticas y de efectividad interpersonal que les permitan comprender las razones que toman las mujeres con estas características, lo cual puede mejorar la calidad de atención que se les brinda. Por otra parte, los profesionales de salud mental, tanto de establecimientos públicos como privados dispondrán de información empírica para implementar estrategias de tratamiento para evaluar y eventualmente modificar los factores psicológicos mantenedores de violencia, así como fortalecer o desarrollar habilidades personales de afrontamiento y redes sociales de apoyo, incluso después de que las mujeres hayan terminado la relación violenta.

\section{Referencias}

Aiquipa, J. J. (2015). Dependencia emocional en mujeres víctimas de violencia de pareja. Revista de Psicología, 33(2), 411-437.

Alsaker, K., Moen, B. E., Baste, V., \& Morken, T. (2016). How has living with intimate partner violence affected the work situation? A qualitative study among abused women in Norway. Journal of family violence, 31(4), 479-487. https://doi.org/10.1007/s10896-016-9806-2

Amor, P. J., \& Echeburúa, E. (2010). Claves psicosociales para la permanencia de la víctima en una relación de maltrato. Clínica Contemporánea, 1(2), 97-104. https://doi.org/10.5093/cc2010v1n2a3

Ballester, A., \& Villanueva, L. (2014). Valoración psicológica en delitos de violencia de género mediante el Inventario Clínico y Multiaxial de Millon III (MCMI-III). Anuario de Psicología Jurídica, 24(1), 9-18. https://doi.org/10.1016/j. apj.2014.06.001

Bardales, O. T. (2014). Lineamientos éticos para las investigaciones en violencia familiary sexual. Lima: Ministerio de la Mujer y Población Vulnerable.

Berger, R. (2015). Now I see it, now I don't: Researcher's position and reflexivity in qualitative research. Qualitative research, 15(2), 219-234. https://doi.org/10.1177/1468794112468475

Buesa, S., \& Calvete, E. (2013). Violencia contra la mujer y síntomas de depresión y estrés postraumático: el papel del apoyo social. International Journal of Psychology and Psychological Therapy, 13(1), 31-45.

Castelló, J. (2012). La superación de la dependencia emocional. Málaga: Ediciones Corona Borealis.

Colegio de Psicólogos del Perú (2018). Código de Ética y Deontología. Recuperado de http://www. cpsp.pe/prueba/images/documentos/otros/codigo_de_eticay_deontologia_cpsp_cdn.pdf 
Flasch, P., Murray, C. E., \& Crowe, A. (2017). Overcoming abuse: A phenomenological investigation of the journey to recovery from past intimate partner violence. Journal of Interpersonal Violence, 32(22), 3373-3401. https://doi. org $/ 10.1177 / 0886260515599161$

Fleming, C. J., \& Resick, P. A. (2017). Help-seeking behavior in survivors of intimate partner violence: Toward an integrated behavioral model of individual factors. Violence and victims, 32(2), 195-209. https://doi.org/10.1891/0886-6708. VV-D-15-00065

Flick, U. (2014). La gestión de la calidad en investigación cualitativa. Madrid: Morata.

Flick, U. (2015). El diseño de investigación cualitativa. Madrid: Morata.

Glaser, B.G., \& Strauss, A.L. (1967). The discovery of grounded theory: Strategies for qualitative research. New York: Aldine.

Gilbert, S. E., \& Gordon, K. C. (2017). Predicting forgiveness in women experiencing intimate partner violence. Violence Against Women, 23(4), 452468. https://doi.org/10.1177/1077801216644071

Goodman, L. A., \& Smyth, K. F. (2011). A call for a social network-oriented approach to services for survivors of intimate partner violence. Psychology of Violence, 1(2), 79-92. https://doi.org/10.1037/ a0022977

Hayati, E. L., Eriksson, M., Hakimi, M., Högberg, U., \& Emmelin, M. (2013). 'Elastic band strategy': Women's lived experiences of coping with domestic violence in rural Indonesia. Global Health Action, 6(7). https://doi.org/10.3402/ gha.v6i0.18894

Heise, L., \& García-Moreno, C. (2003). La violencia en la pareja. En E. G. Krug et al. (Eds.), Informe mundial sobre la violencia y la salud, (pp. 95-131). Washington, D.C.: Organización Panamericana de la Salud.

Huerta, R., Ramírez, N., Ramos, J., Murillo, L., Falcón, C., Misare, M., \& Sánchez, J. (2016). Esquemas cognitivos disfuncionales y dependencia emocional en mujeres con y sin vio- lencia en la relación de pareja de la ciudad de Lima. Revista de Investigación en Psicología, 19(2), 145-162. https://doi.org/10.15381/rinvp. v19i2.12895

Instituto Nacional de Estadística e Informática. (2017). Perú: Encuesta Demográfica y de Salud Familiar 2016 - Nacional y Regional. Lima: Autor.

Jiménez, M., Blanco, J., Medina, S., \& Gómez, R. (2013). Ruta crítica de las mujeres afectadas por violencia de pareja en Venezuela. Recuperado de http://venezuela.unfpa.org/sites/default/ files/pubpdf/RutaCriticaMujeres2013.pdf

Malterud, K., Siersma, V. D., \& Guassora, A. D. (2016). Sample size in qualitative interview studies: Guided by information power. Qualitative Health Research, 26(13), 1753-1760. https:// doi.org/10.1177/1049732315617444

Matheson, F. I., Daoud, N., Hamilton-Wright, S., Borenstein, H., Pedersen, C., \& O'Campo, P. (2015). Where did she go? The transformation of self-esteem, self-identity, and mental well-being among women who have experienced intimate partner violence. Women's Health Issues, 25(5), 561-569. https://doi. org/10.1016/j.whi.2015.04.006

Messing, J. T., Mohr, R., \& Durfee, A. (2015). Intimate partner violence and women's experiences of grief. Child \& Family Social Work, 20(1), 30-39. https://doi.org/10.1111/cfs.12051

Miller, J., \& Glassner, B. (2016). The 'inside' and the 'outside': Finding realities in interviews. En D. Silverman (Ed.), Qualitative Research (pp. 51-66). Londres: Sage Publications.

Molina, J. E., \& Moreno, J. H. (2015). Percepción de la experiencia de violencia doméstica en mujeres víctimas de maltrato de pareja. Universitas Psychologica, 14(3), 997-1008.

Montero, I., Martín-Baena, D., Escribà-Agüir, V., Vives-Cases, C., \& Ruiz-Pérez, I. (2015). Factors associated with the cessation of intimate partner violence in women attending primary care in Spain. Journal of Family Violence, 30(4), 453460. https://doi.org/10.1007/s10896-015-9684-z 
Nóblega, M., \& Muñoz, P. V. (2009). Una aproximación cualitativa a la violencia hacia la mujer en un asentamiento humano de Villa El Salvador. Liberabit, 15(2), 95-108.

Ocampo, L. E. (2015). Autoestima y adaptación en víctimas de maltrato psicológico por parte de la pareja. Psicología desde el Caribe, 32(1), 145168. https://doi.org/10.14482/psdc.32.1.5204

Organización Panamericana de la Salud (2013). Comprender y abordar la violencia contra las mujeres. Violencia infligida por la pareja. Recuperado de https://apps.who.int/ iris/bitstream/handle/10665/98816/WHO_ RHR_12.36_spa.pdf;jsessionid=F9B33B492F4C644F73962530A424A102? sequence $=1$

Organización Panamericana de la Salud. (2014). Violencia contra la mujer en América Latina y el Caribe: análisis comparativo de datos poblacionales de 12 países. Washington D. C.: Autor.

Puente-Martínez, A., Ubillos-Landa, S., Echeburúa, E., \& Páez-Rovira, D. (2016). Factores de riesgo asociados a la violencia sufrida por la mujer en la pareja: una revisión de metaanálisis y estudios recientes. Anales de Psicología, 32(1), 295-306. https://doi.org/10.6018/analesps.32.1.189161

Rapley, T. (2016). Some pragmatics of qualitative data analysis. En D. Silverman (Ed.), Qualitative research (pp. 331-346). Londres: Sage Publications.

Reich, C. M., Jones, J. M., Woodward, M. J., Blackwell, N., Lindsey, L. D., \& Beck, J. G. (2015). Does self-blame moderate psychological adjustment following intimate partner violence? Journal of Interpersonal Violence, 30(9), 1-18. https://doi.org/10.1177/0886260514540800

Rasool, S. (2016). Help-seeking after domestic violence: The critical role of children. Journal of Interpersonal Violence, 31(9), 1661-1686. https://doi.org/10.1177/0886260515569057

Shanthakumari, R. S., Chandra, P. S., Riazantseva, E., \& Stewart, D. E. (2014). 'Difficulties come to humans and not trees and they need to be faced': A study on resilience among Indian women experiencing intimate partner violence. International Journal of Social Psychiatry, 60(7), 703-710. https://doi. org/10.1177/0020764013513440

Silverman, D. (2015). Interpreting qualitative data. Londres: Sage Publications.

Tirone, V., Shorey, R. C., Nathanson, A. M., \& Rhatigan, D. L. (2014). The effect of self-efficacy on the association between social support and relationship alternatives among female intimate partner violence victims. Partner Abuse, 5(3), 279-296. https://doi.org/10.1891/19466560.5.3.279

United Nations. (2015). The world's women 2015: Trends and statistics. New York: Autor.

Walker, L. E. (2017). The battered woman sindrome. New York: Springer Publishing Company.

Watkins, L., Jaffe, A., Hoffman, L., Gratz, K., Messman-Moore, T., \& DiLillo, D. (2014). The longitudinal impact of intimate partner aggression and relationship status on women's physical health and depression symptoms. Journal of Family Psychology, 28(5), 655-665.

World Bank Group (2015). Women, business and the law 2016: Getting to equal. Washington, DC: World Bank.

Zalapa, E. E., García, Y. E., \& Figueroa, G. E. (2012). Resiliencia en mujeres morelianas separadas por violencia conyugal. Revista de Psicología, 14(2), 164-177. 